\title{
Tendência temporal e fatores associados ao consumo de carnes gordurosas na população brasileira entre de 2007 a 2014
}

\author{
Temporal trend and factors associated with consumption of fatty \\ meats by the Brazilian population between 2007 and 2014
}

\author{
Giovana Longo-Silva ${ }^{1}$ \\ Jonas Augusto Cardoso da Silveira ${ }^{1}$ \\ Risia Cristina Egito de Menezes ${ }^{1}$ \\ Patrícia Menezes Marinho ${ }^{1}$ \\ Silvany Barros Olímpio Epifânio ${ }^{1}$ \\ Karine Maria de Melo Brebal ${ }^{1}$ \\ Maysa Helena de Aguiar Toloni ${ }^{2}$
}

${ }^{1}$ Faculdade de Nutrição, Universidade Federal de Alagoas. Av. Lourival Melo Mota s/n, Tabuleiro dos Martins. 57072-900 Maceió AL Brasil.giovana_longo@ yahoo.com.br

${ }^{2}$ Departamento de Nutrição da Universidade Federal de Lavras. Lavras MG Brasil.

\begin{abstract}
The scope of this article is to analyze the temporal trend of the consumption of fatty meats and factors associated with the consumption thereof between 2007 and 2014. A time series of fatty meat consumption (red meat and chicken) was conducted by the "Surveillance of Risk and Protection Factors for Chronic Diseases (Vigitel) telephone survey." The consumption trend was analyzed by joinpoint regression and expressed in annual percentage variation (VPA [95\% CI]), while sociodemographic and behavioral factors were associated in 2007 and 2014 by the generalized linear (log-binomial) model. Between 2007 and 2014, white meat consumption $\geq 5 x /$ week increased (VPA 6.9\% [5.7, 8.1]), while red meat consumption remained stable. Fatty meat consumption showed a significant reduction as of 2011 (VPA -4\% [-7.5; -0.2]), probably due to the reduction in the consumption of fatty red meats. It was found that males, of younger age groups living in the center-west/south-east/south regions, with low education level and alcohol and tobacco abuse were associated with fatty meat consumption; while watching television $>3$ hours/day was only associated in 2014. The consumption of fatty meats showed a reduction trend in the Brazilian population, and its consumption was associated with sociodemographic and behavioral variables. Key words Red meat, Food consumption, Population surveys, Brazil
\end{abstract}

Resumo O objetivo deste artigo é analisar a tendência temporal do consumo de carnes gordurosas (CG) e fatores associados ao seu consumo habitual entre 2007 e 2014. Série temporal do consumo de CG (vermelha e frango/galinha) realizada a partir do inquérito telefônico "Vigilância de Fatores de Risco e Proteção para Doenças Crônicas" (Vigitel). A tendência do consumo foi analisada por regressão "joinpoint” e expressa em variação percentual anual (VPA[IC95\%]), enquanto os fatores sociodemográficos e comportamentais associados em 2007 e 2014 por modelo linear generalizado (log-binomial). Entre 2007-2014, o consumo $\geq 5 x /$ semana de carnes brancas aumentou (VPA $6,9 \%[5,7 ; 8,1])$, enquanto as vermelhas permaneceu estável. O consumo CG apresentou redução significativa a partir de 2011 (VPA -4,0\%[-7,5; -0,2]), provavelmente em função da redução no consumo das vermelhas gordurosas. Identificamos que o sexo masculino, as faixas etárias mais jovens, residir nas regiões $C O / S E / S$, escolaridade $\leq$ 8 anos e o uso abusivo de álcool e tabaco se associaram com o consumo de CG; assistir televisão > 3h/dia foi associada apenas em 2014. No período analisado houve redução no consumo de CG pela população brasileira, sendo seu consumo associado a fatores sociodemográficas e outros comportamentos promotores de DCNT.

Palavras-chave Carne, Consumo de alimentos, Inquéritos populacionais, Brasil 


\section{Introdução}

As dietas ocidentais se caracterizam por consumo elevado de produtos de origem animal, com consequente aporte de gordura saturada e de carnes em quantidades que excedem as recomendações dietéticas ${ }^{1-3}$.

Apesar de serem fontes de proteína de alto valor biológico e possuírem teor elevado de micronutrientes, especialmente ferro, zinco e vitamina $\mathrm{B} 12$, as carnes tendem a ser ricas em gorduras, especialmente saturadas, e quando consumidas em excesso representam risco para o incremento de doenças crônicas não transmissíveis (DCNT) $)^{4,5}$.

Portanto, diante do possível comprometimento da saúde da população ${ }^{5}$ tem-se recomendado temperança no seu consumo, especialmente de carnes gordurosas, submetidas a processamentos industriais ${ }^{6,7}$.

Embora a gordura esteja naturalmente presente nas carnes, estas são consideradas gordurosas quando a quantidade deste macronutriente excede $50 \%$ do seu valor calórico total ${ }^{8}$, incluindo-se nesta classificação as carnes vermelhas (bovinas, suínas, caprinas) além das aves, nas quais a maior concentração de gordura está na pele 9 .

Por esta razão, recomenda-se a remoção da gordura aparente e da pele (aves) de todos os tipos de carne antes do preparo, para evitar sua penetração no interior do alimento durante a cocção $0^{9,10}$. Esta prática consiste em uma das estratégias para redução da mortalidade relacionada às $\mathrm{DCNT}^{9}$, responsáveis por $68 \%$ das mortes no mundo, das quais três quartos, equivalente a 28 milhões de mortes, ocorrem em países de baixo e médio desenvolvimento ${ }^{11}$.

Para o monitoramento anual dos principais fatores de risco e proteção das DCNT no Brasil, o Ministério da Saúde iniciou em 2006, o Sistema de Vigilância de Fatores de Risco e Proteção para Doenças Crônicas por Inquérito Telefônico (Vigitel), realizado de forma contínua desde então ${ }^{12}$, o qual objetiva monitorar por inquérito telefônico a frequência e distribuição dos principais determinantes das DCNT ${ }^{10,12}$. Dentre as investigações relacionadas à alimentação, incluise a frequência do consumo de carnes vermelhas, de frango/galinha e o hábito de consumi-las com gordura.

Diante deste contexto, o objetivo deste estudo foi analisar a tendência temporal do consumo de carnes gordurosas e fatores associados ao consumo excessivo nas capitais brasileiras e no Distrito Federal entre os anos de 2007 e 2014, por meio da análise secundária dos dados do Vigitel.

\section{Métodos}

\section{Delineamento e população de estudo}

O Vigitel é uma pesquisa transversal, realizada por meio telefônico, que possui representatividade de todas as capitais brasileiras e Distrito Federal. Para o presente artigo, compilou-se os dados referentes aos anos de 2007 até 2014 a fim de se analisar alterações temporais no padrão de consumo de carnes pela população adulta brasileira.

Trata-se de uma pesquisa com amostragem probabilística complexa realizada em duas etapas: 1. Sorteio sistemático e estratificado por código de endereçamento postal (CEP), a partir do cadastro eletrônico de linhas residenciais fixas das empresas telefônicas, de, no mínimo, 5 mil linhas telefônicas, as quais foram posteriormente ressorteadas e divididas em réplicas de 200 linhas, reproduzindo a mesma proporção de linhas por CEP do cadastro original. 2. Após identificação das linhas telefônicas elegíveis, foi realizado sorteio de um dos adultos residentes no domicílio. Não foram elegíveis as linhas que: corresponderam a empresas, não mais existiam ou se encontravam fora de serviço, e as que não responderam a seis tentativas de chamadas telefônicas em dias e horários diferentes, incluindo finais de semana e períodos noturnos.

Foi estabelecido tamanho amostral mínimo de aproximadamente 1.500 indivíduos em cada município para estimar, com coeficiente de confiança de $95 \%$ e erro máximo de cerca de três pontos percentuais, a frequência de qualquer fator de risco na população adulta. Erros máximos de cerca de quatro pontos percentuais foram esperados para estimativas específicas, segundo sexo, assumindo-se proporções semelhantes de homens e mulheres na amostra. A reprodução do mesmo processo amostral em todas as edições garante a representatividade espacial e temporal da pesquisa.

Para fins deste estudo, a população elegível foi composta por adultos maiores de 18 anos, participantes do Vigitel nas 26 capitais do Brasil e no Distrito Federal, totalizando uma amostra de $54.249,54.348,54.367,54.339,54.137,45.448$, 52.929 e 40.853 indivíduos nos anos de 2007, 2008, 2009, 2010, 2011, 2012, 2013 e 2014, respectivamente.

\section{Coleta de dados}

As entrevistas telefônicas foram realizadas entre os meses de fevereiro e dezembro de cada 
ano, por uma equipe previamente treinada e supervisionada.

Os questionários eram lidos diretamente na tela de um monitor de vídeo e suas respostas registradas direta e imediatamente em meio eletrônico, permitindo o sorteio automático do membro do domicílio a ser entrevistado, o salto automático de questões não aplicáveis em face de respostas anteriores, a crítica imediata de respostas não válidas e a cronometragem do tempo de duração da entrevista, além de propiciar o abastecimento direto e contínuo no banco de dados do sistema.

\section{Variável dependente}

A variável dependente utilizada nas análises foi o "consumo habitual de carnes gordurosas", obtida a partir das questões: Quando o(a) sr.(a) come carne vermelha (boi, porco, cabrito) com gordura, o(a) sr. (a) costuma: tirar sempre o excesso de gordura, comer com a gordura ou não come carne vermelha com muita gordura? e Quando o(a) sr.(a) come frango/galinha com pele, o(a) sr.(a) costuma: tirar sempre a pele, comer com a pele ou não come pedaços de frango com pele?; destaca-se que esta pergunta se baseia no conteúdo de gordura visível das carnes, uma vez que a percepção e a compreensão de gordura intrínseca pode não ser clara para a população.

$\mathrm{Na}$ análise da série temporal, esta variável foi categorizada em quatro níveis, representando indivíduos que consomem habitualmente (1) carnes vermelhas com gordura e frango/galinha com pele; (2) apenas carnes vermelhas com gorduras; (3) apenas frango/galinha com pele; e, (4) nunca consomem ou retiram o excesso de gordura visível/pele de carnes vermelhas ou frango/galinha. Adicionalmente, também se analisou a evolução da prevalência do consumo predominante $(\geq 5 \mathrm{x} /$ sem.) de carnes vermelhas e frango/galinha pela população brasileira dos sete anos cobertos pelos inquéritos.

Para as análises de fatores associados, o consumo habitual de carnes gordurosas descrito no parágrafo anterior foi transformado numa variável de resposta binária, sendo $Y i=1$ o consumo de carnes vermelhas com gordura e frango/galinha com pele (1), enquanto $Y i=0$, se qualquer outro nível de consumo (2, 3 e 4). Desta forma, buscou-se apontar como risco os indivíduos que possuem padrão de consumo potencialmente deletério para a saúde em relação ao consumo de gorduras saturadas.

\section{Variáveis independentes}

As variáveis independentes foram subdivididas em sociodemográficas (sexo [masculino ou feminino]), idade [18 a 29, 30 a 39, 40 a 49, 50 a 59 ou $\geq 60$ anos], região [norte/nordeste ou centro-oeste/sudeste/sul], situação conjugal [viver ou não com o companheiro], escolaridade $[\leq 8$ anos ou $>8$ anos de estudo] e comportamentais (prática de atividade física [sim ou não], consumo abusivo de álcool [sim ou não], tabagismo [sim ou não], hábito assistir televisão mais de 3 horas/ dia [sim ou não]).

Foi considerado consumo abusivo de álcool cinco ou mais doses (homem) ou quatro ou mais doses (mulher) em uma única ocasião, pelo menos uma vez nos últimos 30 dias, sendo uma dose de bebida alcoólica corresponde a uma lata de cerveja, uma taça de vinho ou uma dose de cachaça, whisky ou qualquer outra bebida alcoólica destilada $^{10}$

Com relação a prática de atividade física foram considerados inativos os que referiram não praticar qualquer atividade física no tempo livre nos últimos três meses e não realizar esforços físicos intensos no trabalho, não se deslocar para o trabalho ou curso/escola caminhando ou de bicicleta perfazendo um mínimo de 20 minutos no percurso de ida e volta e não ser responsável pela limpeza pesada de sua casa ${ }^{10}$

Para a variável tabagismo foi considerado o fumo atual, independentemente do número de cigarros, da frequência e da duração do hábito de fumar.

\section{Análise de dados}

Os bancos de dados utilizados nesta pesquisa são de domínio público e estão disponíveis no endereço: <http://svs.aids.gov.br/bases_vigitel_viva/ $>$. Os procedimentos referentes à união, tratamento e análise dos dados foram realizados no Stata/MP 13.0 (Stata Corp., TX, EUA), sendo que as prevalências do consumo predominante de carne vermelha ou de frango/galinha $(\geq 5 \mathrm{x} /$ sem.) e do consumo habitual de carnes gordurosas foram geradas considerando o plano amostral complexo da pesquisa e o fator de ponderação da amostra.

Adicionalmente, para a análise da série temporal, utilizou-se o software Joinpoint Regression Analysis Software v.4.4.0.0 (The National Cancer Institute, MD, EUA), o qual permite diferenciar padrões lineares na distribuição das estimativas 
de interesse ao longo de dois ou mais intervalos de tempo, identificando segmentos que apresentam tendências específicas em termos de variação percentual anual (VPA), a qual é a média das variações percentuais relativas entre cada um dos sete intervalos de tempo. Nesta análise, a hipótese nula é dada por uma equação linear, enquanto a alternativa se refere a um modelo linear com dois ou mais segmentos que explique melhor a variabilidade dos dados; caso a hipótese nula seja rejeitada, o algoritmo do software continuará incluindo novos segmentos comparando o modelo que apresenta o melhor ajuste dos dados. Para esta comparação, o método de seleção dos modelos foi o teste de permutação pelo método de Monte Carlo, assumindo nível de significância de $5 \%$ e reproduzindo 9.999 replicações ${ }^{13}$.

A fim de identificar se houve alteração nos fatores associados com o consumo habitual de carnes gordurosas no intervalo entre os inquéritos (2007 e 2014), foram construídos dois modelos lineares generalizados múltiplos (GLM, família de ligação binomial e função de ligação $\log$ ), sendo a associação expressa em razão de prevalências (RP), com seus respectivos intervalos de confiança de 99\% (IC99\%). Uma vez que a permanência das variáveis nos modelos finais, bem como sua interpretação, foi baseada no IC, ao invés do valor de $\mathrm{p}^{14}$, optou-se pela utilização do IC99\% como estratégia para gerar estimativas de variabilidade mais conservadoras, dado que o tamanho amostral do presente inquérito pode apontar diferenças estatisticamente significativas mesmo quando estas apresentam baixa magnitude de efeito e/ou sem relevância biológica.

$\mathrm{O}$ processo de modelagem para os inquéritos de 2007 e 2014 foi realizado de forma hierarquizada ("Modelo 1": variáveis sociodemográficas; "Modelo 2": variáveis comportamentais ajustadas pelo "Modelo 1"), considerando-se como elegíveis para compor os modelos múltiplos as variáveis que apresentaram $p<0,20$ na análise univariada. A introdução das variáveis para composição dos modelos finais foi por meio do método stepwise forward, permanecendo nos modelos apenas aquelas variáveis em que o IC99\% não incluiu o 1,00; todavia, caso alguma variável do "Modelo 1" sofresse alteração em relação a sua associação com o consumo habitual de carnes gordurosas durante a composição do "Modelo 2", esta permanecia como variável de ajuste.

\section{Aspectos éticos}

Todos os inquéritos referentes ao Vigitel foram aprovados pelo Comitê Nacional de Ética em Pesquisa com Seres Humanos do Ministério da Saúde. Todavia, por se tratar de uma análise secundária de dados de domínio público, nova aprovação por comitê de ética em pesquisa se faz desnecessária.

\section{Resultados}

No período entre 2007 e 2014 não houveram alterações significativas na distribuição etária e de sexo da população brasileira, bem como o percentual de residentes dentre as cinco macrorregiões permaneceram estáveis. Em relação a outras características da população, a proporção de indivíduos que declararam não estar trabalhando na ocasião da pesquisa (2007: $35,1 \%$; 2014: $36 \%$ ) e apresentava escolaridade $\leq 8$ anos (2007: 68,4\%; 2014: 66,4\%) se mantiveram relativamente estáveis.

Quanto à variação do consumo predominante de carnes (Figura 1), identificou-se que o aumento ocorrido no intervalo analisado foi devido ao aumento de $\sim 6$ pp no consumo de carne de frango ou galinha na população, o que representou VPA de 6,9\% (5,7; 8,1 IC95\%); o consumo predominante de carnes vermelhas permaneceu estável.

Em relação ao consumo habitual de carnes gordurosas (Figura 2), nota-se uma tendência de redução provavelmente em função do consumo oriundo das carnes vermelhas. Tal observação é baseada na manutenção da prevalência de indivíduos que consomem carne de frango ou galinha com pele entre o período de 2007 e 2014 (VPA $=0,2 \%$ [-1,0; 1,4 IC95\%]) e redução expressiva na prevalência de consumo de carnes vermelhas com gordura a partir do ano de 2011 (VPA = $-4,0 \%$ [-7,5; -0,2 IC95\%]). Desta forma, é plausível considerar que a redução linear identificada entre indivíduos que consomem os dois tipos de carnes com gordura, pode ter sido predominantemente devido à redução no consumo de gordura proveniente das carnes vermelhas.

$\mathrm{Na}$ Tabela 1 são descritas as prevalências do consumo habitual de carnes vermelhas e de frango/galinha com gordura para cada ano analisado com suas respectivas VPA de cada uma das variáveis independentes selecionadas. De modo geral, todas as categorias examinadas seguiram padrão similar de redução do consumo, todavia, desta- 


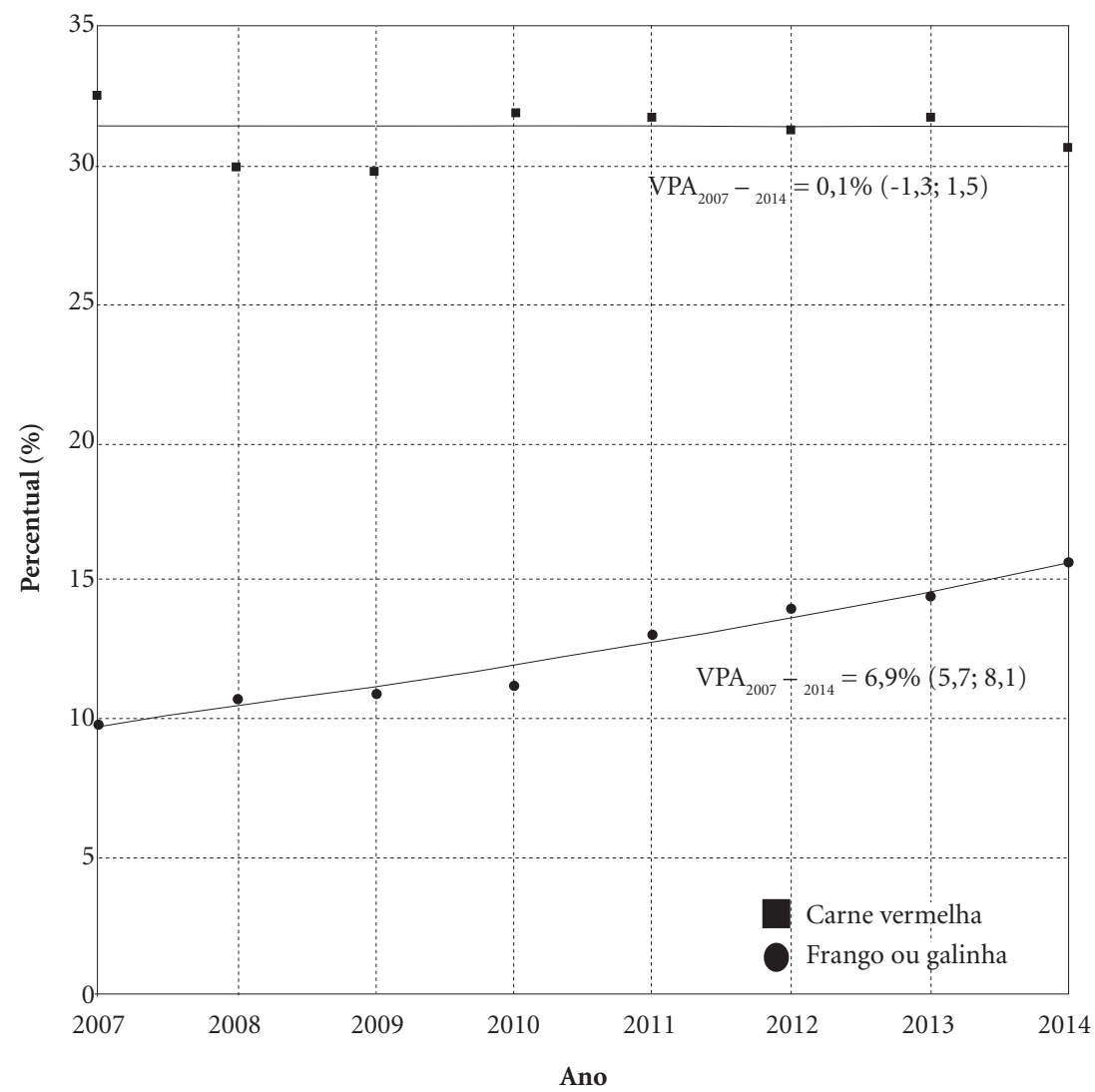

Figura 1. Variação do consumo predominante ( $\geq 5 \mathrm{x} /$ semana) de carnes vermelhas e frango ou galinha pela população brasileira. Vigitel, 2014.

VPA - Variação percentual anual do segmento analisado, expressa em percentual com seu respectivo intervalo de confiança de $95 \%$.

ca-se as diferenças socioeconômicas - expressas pela categorização das macrorregiões - quanto a redução do consumo exclusiva nas regiões $\mathrm{S} / \mathrm{SE} /$ $\mathrm{CO}$, praticamente anulando a diferença de $5,7 \mathrm{pp}$ entre as regiões em 2007.

Em relação à faixa etária, observa-se que há redução da prevalência do consumo habitual de carnes gordurosas em função do envelhecimento da população, independentemente do ano analisado. Outro ponto digno de nota se refere ao grupo etário dos indivíduos entre 50-59 anos de idade, que apresentaram VPA de -7,0\% (-10,8; -3,0 IC95\%); contudo, a prevalência de consumo de carnes gordurosas dentre a parcela da população mais jovem (18 - 29 anos) permaneceu não apenas estável no intervalo analisado, mas também a maior dentre os estratos etários.

Ainda nas variáveis sociodemográficas, identificamos que em 2007 havia uma relação inversa entre escolaridade e a prevalência de consumo de carnes gordurosas. Porém, em 2014, essa relação desaparece devido à manutenção da prevalência dentre os mais escolarizados, concomitante à redução de 3,5\% (-5,3; -1,7 IC95\%) observada entre os indivíduos com tempo $\leq 8$ anos de estudo.

Dentre as variáveis comportamentais é interessante notar o potencial de agrupamento de fatores de risco para DCV que ocorre entre consumo de carnes com gordura e o consumo abusivo e o tabagismo. Para ambos, a diferença de riscos observada em 2007 se mantém estável ou até se amplia em 2014.

Nas Tabelas 2 e 3, apresentamos os modelos GLM múltiplos para o ano de 2007 e 2014 . Nestas análises, foi possível perceber que houve uma atenuação na magnitude do efeito das razões de prevalência para todas as variáveis analisadas, com exceção do tabagismo (2007: 1,36 [1,19; 1,57 IC95\%] / 2014: 1,76 (1,27; 2,45 IC95\%]). Além disso, o hábito de assistir $\mathrm{TV} \geq 3$ horas/dia que não se associou ao desfecho na análise univariada em 2007, permaneceu associada com o consumo 


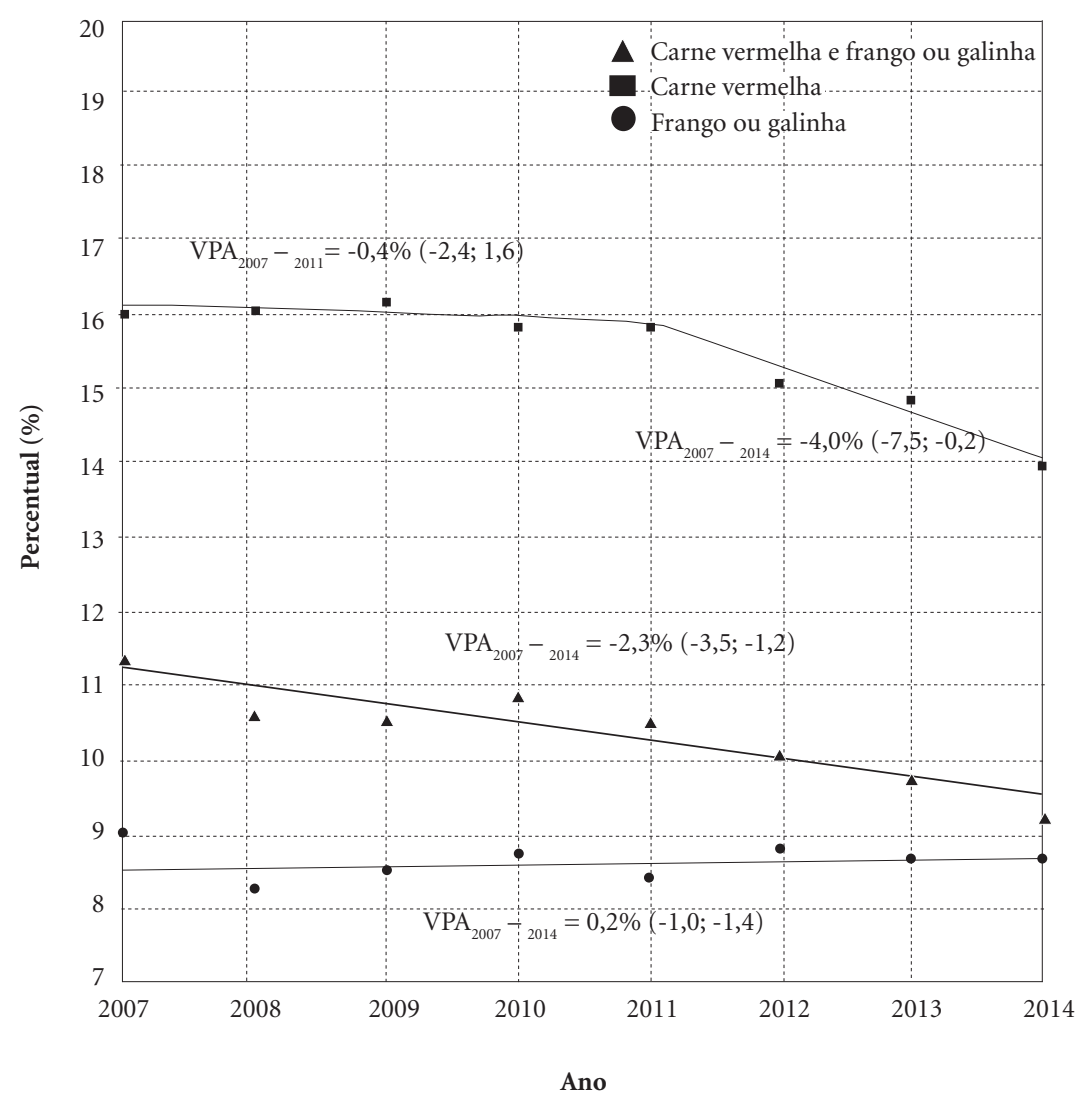

Figura 2. Variação do consumo habitual de carnes gordurosas pela população brasileira. Vigitel, 2014.

VPA - Variação percentual anual do segmento analisado, expressa em percentual com seu respectivo intervalo de confiança de $95 \%$.

de carnes gordurosas no modelo múltiplo final em 2014.

\section{Discussão}

Os dados apresentados indicam que o consumo predominante de carnes vermelhas se manteve estável no decorrer de oito anos na população adulta brasileira residentes nas capitais e no Distrito Federal, enquanto que o consumo de carne de frango ou galinha apresentou tendência ascendente.

Um estudo de série temporal com adultos brasileiros $^{15}$ identificou aumento no consumo de carne frango na década de 70. Posteriormente, Marinho et al. ${ }^{16}$ ao investigarem as práticas e mudanças alimentares na população de Brasília, Distrito Federal, constataram que esse tipo de carne apresentou maior prevalência de consumo semanal em relação à carne vermelha.

Destaca-se que, as análises temporais do nosso estudo demonstraram uma redução do consumo de carnes gordurosas pela população brasileira, o que pode ser atribuído, especialmente, à diminuição significativa na prevalência de indivíduos que referiram comer habitualmente carne vermelha com gordura.

Apesar disso, outros estudos conduzidos no país demonstram que o consumo de carnes gordurosas ainda é elevado entre os brasileiros, como observado no Inquérito Domiciliar sobre Comportamentos de Risco e Morbidade Referida de Doenças e Agravos não Transmissíveis realizado pelo Ministério da Saúde em 15 Capitais brasileiras e Distrito Federal, onde se identificou que o percentual de indivíduos que relatou nunca, quase nunca ou só algumas vezes retirar a gordu- 


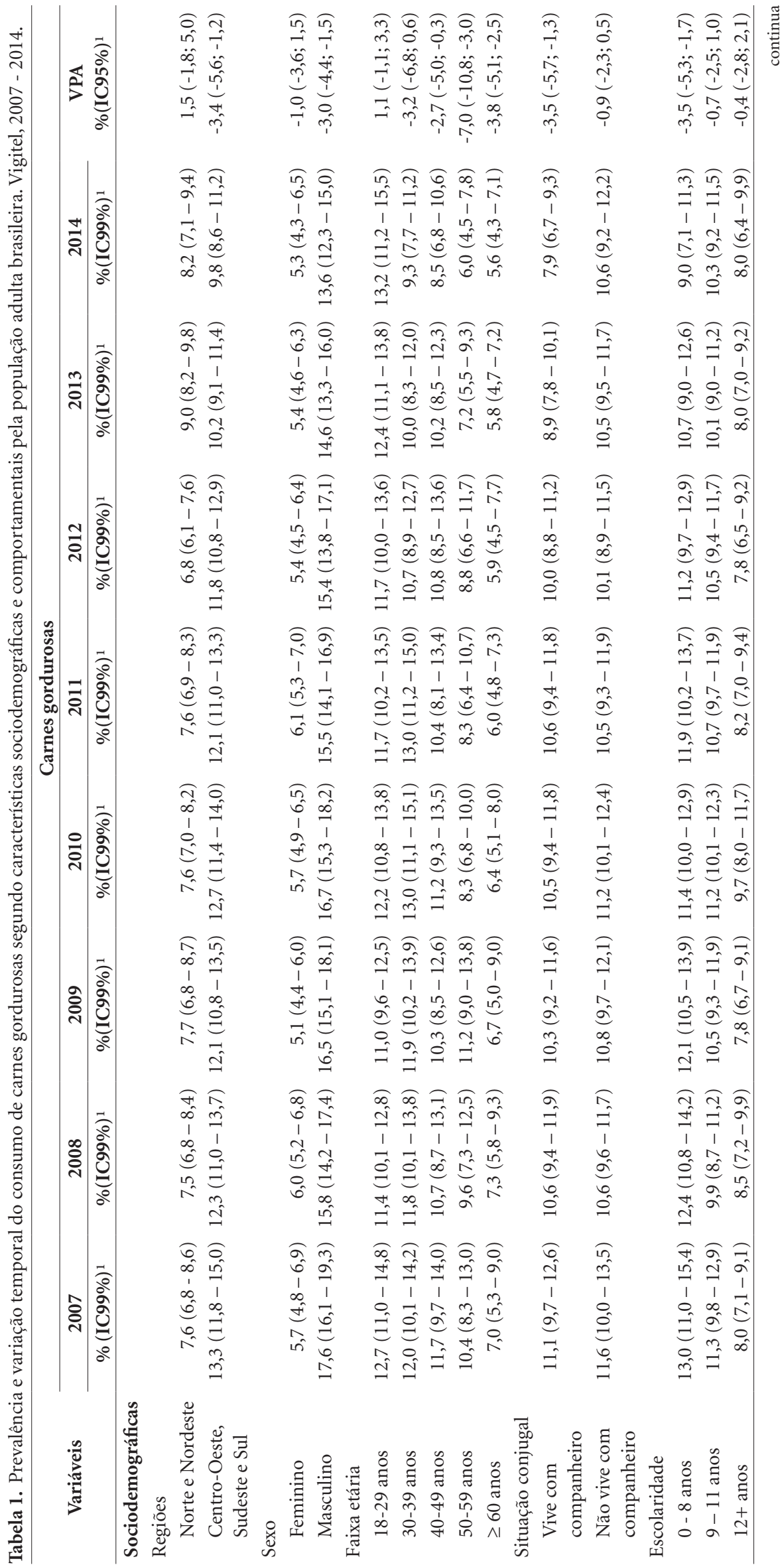




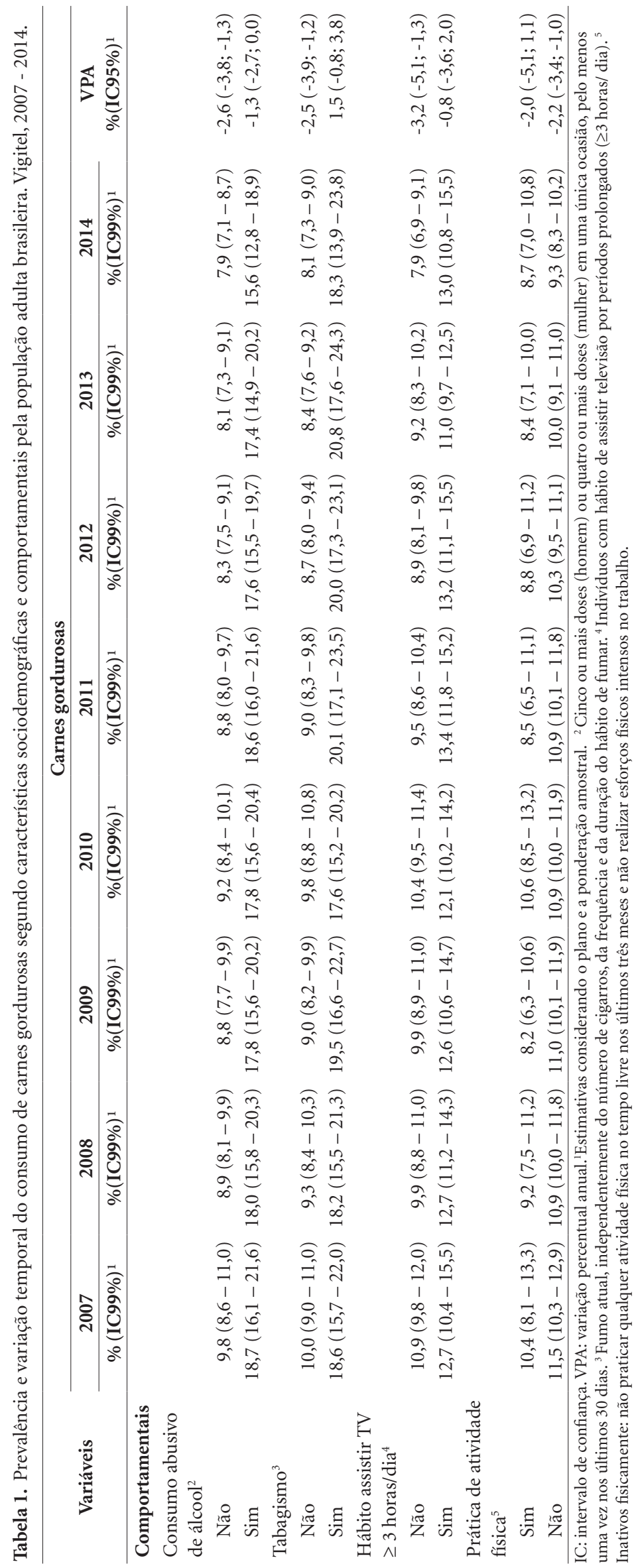


Tabela 2. Fatores socioedemográficos e comportamentais associados com o consumo de carnes gordurosas pela população adulta brasileira. Vigitel, 2007.

\begin{tabular}{|c|c|c|c|c|c|}
\hline \multirow[b]{2}{*}{ Variáveis } & \multirow[b]{2}{*}{$\mathrm{RP}^{1}$ bruta (IC99\%) } & \multicolumn{2}{|l|}{ Modelo 1} & \multicolumn{2}{|l|}{ Modelo 2} \\
\hline & & $\begin{array}{l}\mathrm{RP}^{1} \text { ajustada } \\
\quad(\mathrm{IC} 99 \%)\end{array}$ & $\mathbf{p}$ & $\begin{array}{l}\mathrm{RP}^{1} \text { ajustada } \\
\quad(\mathrm{IC} 99 \%)\end{array}$ & $\mathrm{p}$ \\
\hline \multicolumn{6}{|l|}{ Sociodemográficas } \\
\hline \multicolumn{6}{|l|}{ Regiões } \\
\hline $\begin{array}{l}\text { Centro-Oeste, } \\
\text { Sudeste e Sul }\end{array}$ & $1,74(1,47-2,06)$ & $1,83(1,54-2,17)$ & $<0,001$ & $1,81(1,53-2,14)$ & $<0,001$ \\
\hline Norte e Nordeste & 1 & 1 & & 1 & \\
\hline \multicolumn{6}{|l|}{ Sexo } \\
\hline Masculino & $3,07(2,61-3,61)$ & $2,99(2,52-3,54)$ & $<0,001$ & $2,75(2,29-3,31)$ & $<0,001$ \\
\hline Feminino & 1 & 1 & & 1 & \\
\hline \multicolumn{6}{|l|}{ Faixa etária (anos) } \\
\hline $18-29$ anos & $1,83(1,34-2,50)$ & $2,21(1,60-3,06)$ & $<0,001$ & $1,96(1,39-2,76)$ & $<0,001$ \\
\hline $30-39$ anos & $1,73(1,34-2,22)$ & $1,87(1,45-2,42)$ & $<0,001$ & $1,69(1,28-2,23)$ & $<0,001$ \\
\hline $40-49$ anos & $1,68(1,28-2,19)$ & $1,87(1,39-2,50)$ & $<0,001$ & $1,71(1,25-2,33)$ & $<0,001$ \\
\hline $50-59$ anos & $1,49(1,06-2,10)$ & $1,67(1,18-2,36)$ & $<0,001$ & $1,57(1,11-2,22)$ & 0,001 \\
\hline$\geq 60$ anos & 1 & 1 & & 1 & \\
\hline \multicolumn{6}{|l|}{ Escolaridade (anos) } \\
\hline $0-8$ anos & $1,62(1,32-1,98), 62(1,32$ & $1,93(1,54-2,41)$ & $<0,001$ & $1,85(1,51-2,26)$ & $<0,001$ \\
\hline 9-11 anos & $1,40(1,16-1,69)$ & $1,46(1,20-1,78)$ & $<0,001$ & $1,45(1,19-1,75)$ & $<0,001$ \\
\hline $12+$ anos & 1 & 1 & & 1 & \\
\hline \multicolumn{6}{|l|}{ Comportamentais } \\
\hline \multicolumn{6}{|l|}{$\begin{array}{l}\text { Consumo abusivo de } \\
\text { álcool }\end{array}$} \\
\hline Sim & $1,92(1,59-2,31)$ & & & $1,33(1,09-1,63)$ & $<0,001$ \\
\hline Não & 1 & & & 1 & \\
\hline \multicolumn{6}{|l|}{ Tabagismo } \\
\hline Sim & $1,88(1,58-2,21)$ & & & $1,36(1,19-1,57)$ & $<0,001$ \\
\hline Não & 1 & & & 1 & \\
\hline \multicolumn{6}{|l|}{$\begin{array}{l}\text { Hábito assistir } \mathrm{TV} \geq 3 \\
\text { horas/dia }\end{array}$} \\
\hline Sim & $1,17(0,95-1,45)$ & & & & \\
\hline Não & 1 & & & & \\
\hline \multicolumn{6}{|l|}{$\begin{array}{l}\text { Prática de atividade } \\
\text { física }\end{array}$} \\
\hline Não & $1,11(0,83-1,47)$ & & & & \\
\hline Sim & 1 & & & & \\
\hline
\end{tabular}

RP: Razão de prevalência calculada sob ponderações devido ao desenho amostral. IC 99\%: intervalo de 99\% de confiança. ${ }^{1}$ Estimativas considerando o plano e a ponderação amostral. Modelo 1: $\mathrm{N}=40254$ - ajustado pelas regiões, sexo, faixa etária e escolaridade. Modelo 2: N = 40254- ajustado pelo Modelo 1.

ra visível da carne ou a pele do frango variou de $48 \%$ a $49,3 \%$ nas diferentes faixas etárias e regiões do País ${ }^{17}$. Corroborando tal asserção, a partir de dados da Pesquisa Nacional de Saúde (PNS) de $2013^{18}$, constatou-se que $37,2 \%$ da população brasileira relatou consumo de carne ou frango com excesso de gordura. Tais achados, em adição à presença de outros alimentos não saudáveis tidos como fatores de risco para DCNT, refletem à grande prevalência de consumo de alimentosfonte de gordura saturada, visto que 4 a cada 10 brasileiros referiram consumir carne ou frango com excesso de gordura.

Diante da redução nas prevalências de consumo de carne gordurosa identificada em nosso estudo, cabe referir que, apesar de o teor de gordura da carne bovina consistir em aspecto importante na escolha dos consumidores, sendo a 
Tabela 3. Fatores socioedemográficos e comportamentais associados com o consumo de carnes gordurosas pela população adulta brasileira. Vigitel, 2014.

\begin{tabular}{|c|c|c|c|c|c|}
\hline \multirow[b]{2}{*}{ Variáveis } & \multirow[b]{2}{*}{$\begin{array}{l}\text { RP' bruta } \\
(\text { IC99\% })^{2}\end{array}$} & \multicolumn{2}{|l|}{ Modelo 1} & \multicolumn{2}{|l|}{ Modelo 2} \\
\hline & & $\begin{array}{l}\text { RP1 ajustada } \\
(\text { IC } 99 \%)^{2}\end{array}$ & $\mathbf{p}$ & $\begin{array}{l}\text { RP1 ajustada } \\
(\text { IC } 99 \%)^{2}\end{array}$ & $\mathbf{p}$ \\
\hline \multicolumn{6}{|l|}{ Sociodemográficas } \\
\hline \multicolumn{6}{|l|}{ Regiões } \\
\hline Centro-Oeste, Sudeste e Sul & $1,20(0,99-1,46)$ & $1,23(1,01-1,50)$ & 0,006 & $1,20(0,99-1,47)$ & 0,015 \\
\hline Norte e Nordeste & 1 & 1 & & 1 & \\
\hline \multicolumn{6}{|l|}{ Sexo } \\
\hline Masculino & $2,56(2,10-3,12)$ & $2,42(1,96-3,00)$ & 0,000 & $2,21(1,75-2,78)$ & $<0,001$ \\
\hline Feminino & 1 & 1 & & 1 & \\
\hline \multicolumn{6}{|l|}{ Faixa etária (anos) } \\
\hline 18-29 anos & $2,38(1,68-3,36)$ & $2,47(1,71-3,57)$ & $<0,001$ & $2,29(1,60-3,29)$ & $<0,001$ \\
\hline 30-39 anos & $1,67(1,25-2,24)$ & $1,81(1,31-2,50)$ & $<0,000$ & $1,65(1,21-2,25)$ & $<0,001$ \\
\hline 40-49 anos & $1,53(1,13-2,08)$ & $1,56(1,12-2,18)$ & 0,001 & $1,48(1,07-2,09)$ & 0,002 \\
\hline 50-59 anos & $1,08(0,70-1,64)$ & $1,10(0,72-1,67)$ & 0,572 & $1,05(0,70-1,60)$ & 0,744 \\
\hline$\geq 60$ anos & 1 & & & & \\
\hline \multicolumn{6}{|l|}{ Escolaridade (anos) } \\
\hline $0-8$ anos & $1,12(0,79-1,59)$ & $1,43(1,00-2,05)$ & 0,010 & $1,29(0,92-1,81)$ & 0,053 \\
\hline 9-11 anos & $1,29(1,05-1,59)$ & $1,28(1,03-1,61)$ & 0,004 & $1,17(0,94-1,46)$ & 0,061 \\
\hline $12+$ anos & 1 & 1 & & 1 & \\
\hline \multicolumn{6}{|l|}{ Comportamentais } \\
\hline \multicolumn{6}{|l|}{ Consumo abusivo de álcool } \\
\hline Sim & $1,99(1,64-2,41)$ & & & $1,31(1,04-1,66)$ & 0,003 \\
\hline Não & 1 & & & 1 & \\
\hline \multicolumn{6}{|l|}{ Tabagismo } \\
\hline Sim & $2,26(1,69-3,03)$ & & & $1,76(1,27-2,45)$ & $<0,001$ \\
\hline Não & 1 & & & 1 & \\
\hline \multicolumn{6}{|l|}{ Hábito assistir TV $\geq 3$ horas/dia } \\
\hline Sim & $1,63(1,27-2,10)$ & & & $1,49(1,11-1,99)$ & 0,001 \\
\hline Não & 1 & & & 1 & \\
\hline \multicolumn{6}{|l|}{ Prática de atividade física } \\
\hline Não & $1,07(0,85-1,35)$ & & & & \\
\hline Sim & 1 & & & & \\
\hline
\end{tabular}

${ }^{1}$ RP: Razão de prevalência calculada sob ponderações devido ao desenho amostral. ${ }^{2}$ IC $99 \%$ : intervalo de $99 \%$ de confiança calculado sob ponderações devido ao desenho amostral. Modelo 1: $\mathrm{N}=40254$ - ajustado pelas regiões, sexo, faixa etária e escolaridade. Modelo 2: N = 40254- ajustado pelo Modelo 1.

gordura ligeiramente visível preferida em muitos países $^{19,20}$, as preferências por carnes magras parece estar aumentando ${ }^{21}$.

Henchion et al. ${ }^{22}$ apresentaram as estimativas para 2022 do consumo de carne na Europa, constatando que as carnes bovina e ovina deverão representar uma quota de diminuição de consumo, enquanto as carnes brancas, em especial a de aves possui tendência de aumento. Semelhantemente, Ngapo e Dransfield ${ }^{21}$, a partir de levantamentos realizados em 1955, 1982 e 2002, concluíram que os britânicos, embora ainda considerem a gor- dura uma característica importante na seleção das carnes no momento da compra, o nível de gordura preferido pelos consumidores não permaneceu estático, sendo que no último levantamento a maioria preferiu carnes mais magras que os antecessores.

Assim, cada vez mais as evidências sugerem que uma mudança positiva e definitiva está ocorrendo ${ }^{23,24}$, apontando redução do consumo de gordura, especialmente carnes e aumento de grãos integrais, frutas e vegetais. Tal mudança positiva corresponde ao terceiro estágio da tran- 
sição nutricional Behavioural chance, proposto por Popkin ${ }^{24}$ e que pode justificar as tendências temporais constatadas em nosso estudo.

Ao analisarmos o consumo de carnes gordurosas segundo as características sociodemográficas, econômicas e comportamentais nos inquéritos de 2007 e 2014, este mostrou-se estatisticamente associado nos modelos explicativos finais ao sexo masculino, faixa etária de 18 a 29 anos, regiões centro-oeste/sudeste/sul, menor escolaridade ( $\leq 8$ anos de estudo), inatividade física, consumo abusivo de álcool, tabagismo e tempo usual assistindo televisão superior a 3 horas/dia. Ressalta-se que, neste último, a associação com o desfecho em estudo foi observada somente em 2014.

Associações similares às do nosso estudo, referentes à idade e gênero, foram encontradas por Schneider et al. $^{25}$, ao descreverem a frequência do consumo de carnes e o hábito de consumi-las com excesso de gordura, identificaram que, entre adultos com idade igual ou superior a 20 anos, $52,3 \%$ referiram consumo de carne com excesso de gordura. Destes, a maior frequência de consumo foi observado no sexo masculino $(66,7 \%)$, e nos indivíduos com idades mais jovens dentre as categorias de faixa etárias analisadas. Adicionalmente, foi constatado no referido trabalho que os homens apresentaram um consumo cerca de $60 \%$ maior que as mulheres em relação a todas as variáveis de exposição estudadas.

Buscando explorar as potenciais variações das atitudes e motivações para adoção de comportamentos alimentares saudáveis, nas distintas faixas de idade e gênero, Chambers et al. ${ }^{26}$ conduziram uma pesquisa qualitativa no Reino Unido, na qual concluíram que os participantes com idades entre 18 e 30 anos foram os menos propensos a realizar escolhas alimentares com base nas considerações de saúde, priorizando o tempo, forma de preparo e preço. Entre as mulheres, no entanto, as escolhas mais saudáveis foram relacionadas à preocupação com sua aparência.

No tocante da distribuição territorial, identificamos em 2014 estabilidade no consumo de carnes entre as macrorregiões analisadas, embora o percentual de variação entre 2007 e 2014 tenha sido negativo nas regiões centro oeste/sudeste/ sul $(-3,4 \%)$, e positivo nas regiões norte/nordeste $(1,5 \%)$.

Cumpre referir que o Brasil historicamente caracteriza-se por sua polarização social e geográfica, constatando-se disparidades regionais ${ }^{27}$, evidenciadas principalmente pelas regiões norte, nordeste e sul, sudeste, que representam os extremos dos indicadores de saúde ${ }^{28}$.
Embora tais disparidades e iniquidades ainda predominem, o país vivenciou melhorias sociais nos últimos anos. Em consonância com os Objetivos do Desenvolvimento do Milênio ${ }^{29}$, alcançou as metas internacionais e nacionais voltadas para erradicação da pobreza entre os anos de 1990 e 2012. Especificamente nas regiões norte e nordeste a taxa de extrema pobreza reduziu de $28,5 \%$ para $7,3 \%$ e $7,5 \%$ para $3,8 \%$, respectivamente, contribuindo assim para redução da desigualdade intra-país, e consequentemente da insegurança alimentar.

$\mathrm{Na}$ mesma perspectiva, acrescenta-se que a baixa escolaridade, variável proxy da renda fami$\operatorname{liar}^{30}$, mostrou-se associada, após ajuste às demais variáveis, ao consumo das carnes gordurosas.

Apesar de o grupo das carnes corresponder ao item mais caro da alimentação, representa o maior percentual dos gastos das famílias brasileiras, em relação aos demais alimentos. Segundo dados da Pesquisa de Orçamento Familiar (POF) 2008-0931, 15,1\% do gasto das famílias com alimentação é oriundo da carne, vísceras e pescados, sendo o grupo de menor renda o que proporcionalmente mais gasta com a compra desse gênero alimentício. Levy-Costa et al. $^{32}$ apontaram para uma relação direta entre a participação relativa das carnes nas calorias da dieta dos brasileiros com o nível de rendimentos familiares.

Em adição ao maior consumo de produtos de origem animal, tende a prevalecer nas classes de menor rendimento o consumo de gordura, o que pode ser justificado por preferências culturais, menor disponibilidade, acesso e informação para adoção de hábitos saudáveis ${ }^{33}$. Somado a estas questões, Schneider et al. ${ }^{25}$, ressaltam que a falta de incentivo de órgãos públicos de saúde quanto à adoção de atitudes de vida mais saudáveis, pode ser uma das explicações para o consumo de carnes com excesso de gordura predominante entre indivíduos com menor nível de instrução.

Cabe destacar que o consumo de carnes gordurosas associou-se a padrões inadequados, sendo que o tabagismo, consumo abusivo de álcool, inatividade física e maior tempo gasto assistindo televisão permaneceram nos modelos logísticos finais. Esta constatação também foi identificada por Azevedo et al. ${ }^{34}$ ao estudarem comportamentos alimentares de risco para doenças crônicas em Recife, PE.

Sabe-se que as escolhas alimentares são influenciadas por uma complexa rede de fatores, como demográficos, econômicos, culturais e comportamentais. O impacto que estes fatores, especialmente os relacionados a estilo de vida 
não saudável, como hábito tabágico, consumo de álcool e sedentarismo, tem gerado impacto adverso sobre as decisões alimentares, sendo esta afirmativa consistente com a literatura. Nesse contexto, estudos conduzidos em diferentes regiões do mundo têm demonstrado que os indivíduos com esses hábitos de vida são mais propensos a tomarem decisões negativas em relação à sua saúde, incluindo a escolha de alimentos não saudáveis ${ }^{35,36}$.

Nessa premissa, Perrin et al. ${ }^{37}$ utilizando dados de uma pesquisa para monitoramento de tendências e determinantes em doenças cardiovasculares, revelaram que entre homens franceses, com idades entre 45 e 64 anos, a maior aderência ao padrão alimentar saudável ocorreu entre aqueles que praticavam regularmente algum tipo de atividade física e que não tinham o hábito de fumar, evidenciando que tais padrões parecem refletir de forma mais apropriada na prática alimentar saudável da população em estudo.

Adicionalmente, cumpre destacar que os padrões inadequados supracitados, além das dislipidemias associadas principalmente ao consumo excessivo de gorduras animais, estão entre os principais fatores de risco para DCNT, segundo a Organização Mundial da Saúde ${ }^{38}$.

Conforme mencionado anteriormente, a carne integra a composição de uma alimentação adequada e saudável, em virtude de suas propriedades nutricionais, todavia o seu alto consumo e, principalmente, o consumo das gorduras aparentes são sabidamente associados a doenças cardiovasculares e alguns tipos de câncer.

Por esta razão e, em decorrência do alto impacto ambiental gerado pela sua produção, a redução do consumo de carne é central em muitos debates científicos sobre dietas saudáveis e sustentáveis ${ }^{39-41}$.

Neste contexto, Westhoek et al..$^{39}$ testaram os efeitos de dietas com diferentes composições de produtos animais e evidenciaram que a redução do consumo de carne, produtos lácteos e ovos na União Europeia permitiria redução de $40 \%$ nas emissões de nitrogênio, $25-40 \%$ das emissões de gases de efeito estufa e $23 \%$ da utilização de terras agrícolas para produção de alimentos. Ademais, concluíram que a redução em $40 \%$ da ingestão de gordura saturada conduziria a redução da mortalidade cardiovascular.

Nesta perspectiva, o monitoramento contínuo dos fatores de risco de DCNT por meio do Vigitel é de primordial importância, face às metas pactuadas no Plano de Enfrentamento de Doenças Crônicas não transmissíveis com a OMS, voltadas para a redução do tabagismo, consumo de álcool, inatividade física e alimentação inadequada ${ }^{42}$.

Apesar de inquéritos que se baseiam em informações autorreferidas estarem sujeitos a subestimação ou superestimação nos dados, Monteiro et al. ${ }^{43}$, validaram os indicadores de consumo de alimentos e bebidas do Vigitel e não encontraram tendência de superestimação na frequência do consumo de alimentos associados ao risco de doenças, havendo boa reprodutibilidade e adequada validade para a maioria dos indicadores, incluindo os utilizados no presente estudo. Conclusão semelhante foi identificada por Mendes et al. ${ }^{44}$, que ao avaliarem a reprodutibilidade e validade de indicadores de consumo de alimentos e bebidas levantados pelo Vigitel, verificaram que o consumo de carne com gordura ou frango/galinha com pele obteve reprodutibilidade com concordância substancial e ausência de subestimação.

Outra limitação de inquéritos telefônicos refere-se à exclusão dos indivíduos residentes em domicílios sem cobertura por telefonia fixa, sub -amostrando, desta forma, a população de baixa renda. No entanto, a correção do efeito de desenho objetiva minimizar este viés ${ }^{45}$.

Assim, considerando o objetivo aqui proposto é possível concluir que o consumo de carnes gordurosas pelos adultos no Brasil apresentou tendência descendente no período entre os anos de 2007 e 2014; embora este seja um panorama relativamente positivo, por outro lado, identificamos que a maior frequência de consumo se associou com o sexo masculino, inversamente com a faixa etária (sendo os mais jovens os maiores consumidores), residentes das regiões centro-oeste/sudeste/sul e indivíduos com baixo nível de escolaridade ( $\leq 8$ anos de estudo). Adicionalmente, observamos o agrupamento de reconhecidos fatores de risco para DCNT, juntamente com o consumo excessivo de gorduras saturadas, como o uso abusivo de álcool e do tabaco. Por fim, assistir televisão $\geq 3$ horas/dia, considerado aqui como um marcador indireto da inatividade física, se associou com o consumo de carnes gordurosas em 2014, mas não em 2007.

Espera-se que nossos achados possam contribuir para melhor compreensão do problema, de forma a subsidiar a formulação ou aprimoramento de estratégias com abordagens multifatoriais voltadas para a ocorrência de DCNT, destacando a importância de ações de prevenção primária (incluindo a utilização dos meios de comunicação de massa para disseminar informações sobre alimentação saudável) e o papel da atenção básica na saúde da população brasileira. 


\section{Referências}

1. Linseisen J1, Lech AA, Ocké M, Amiano P, Agnoli C, Ferrari P, Sonestedt E, Chajès V, Bueno-de-Mesquita HB, Kaaks R, Weikert C, Dorronsoro M,Rodríguez L, Ermini I, Mattiello A, van der Schouw YT, Manjer J, Nilsson S, Jenab M, Lund E, Brustad M, Halkjaer J, Jakobsen MU, Khaw KT, Crowe F, Georgila C, Misirli G, Niravong M, Touvier M, Bingham S, Riboli E, Slimani N. Dietary fat intake in the European prospective into cancer and nutrition: results from the 24-h dietary recalls. Eur J Clin Nutr 2009; 63(Supl. 4):61-80.

2. Ocké $\mathrm{MC1}$, Larrañaga $\mathrm{N}$, Grioni $\mathrm{S}$, van den Berg SW, Ferrari P, Salvini S, Benetou V, Linseisen J, Wirfält $\mathrm{E}$, Rinaldi $S$, Jenab $M$, Halkjaer J, Jakobsen $M U$, Niravong M, Clavel-Chapelon F, Kaaks R, Bergmann M, Moutsiou E, Trichopoulou A, Lauria C, Sacerdote C, Bueno-de-Mesquita HB, Peeters $\mathrm{PH}$, Hjartåker A, Parr CL, Tormo MJ, Sanchez MJ, Manjer J, Hellstrom V, Mulligan A, Spencer EA, Riboli E, Bingham S, Slimani N. Energy intake and sources of energy intake in the European prospective investigation into cancer and nutrition. Eur J Clin Nutr 2009; 63(Supl. 4):3-15.

3. Pan A, Sun Q, Bernstein AM, Schulze MB, Manson JE, Stampfer MJ, Willett WC, Hu FB. Red meat consumption and mortality: results from 2 prospective cohort studies. Arch Intern Med 2012; 172(7):555-563.

4. Xavier HT, Izar MC, Faria Neto JR, Assad MH, Rocha VZ, Sposito AC, Fonseca FA, Santos JE, Santos RD, Bertolami M C, Faludi AA, Martinez TLR, Diament J, Guimarães A, Forti NA, Moriguchi E, Chagas ACP, Coelho OR, Ramires JAF; Sociedade Brasileira de Cardiologia. V Diretriz Brasileira de Dislipidemias e Prevenção da Aterosclerose. Arq Bras Cardiol 2013. [acessado 2016 Set 07]. Disponível em: http://www.scielo.br/pdf/abc/ v101n4s1/v101n4s1.pdf

5. Ferguson LR. Meat and cancer. Meat Sci 2010; 84(2):308-313.

6. Food and Agriculture Organization of the United Nations (FAO). Livestock's long shadow: environmental issues and options. Rome: FAO; 2006.

7. World Cancer Research Fund; American Institute for Cancer Research (AICR). Food, Nutrition, Physical Activity, and the Prevention of Cancer: a Global Perspective. Washington: AICR; 2007. [acessado 2016 Set 07]. Disponível em: https://www.dietandcancerreport.org/ cancer_resource_center/downloads/Second_Expert_

8. Philippi ST. Nutrição e técnica dietética. $3^{\circ}$ ed. São Paulo: Manole; 2014.

9. Santos RD, Gagliardi ACM, Xavier HT, Magnoni CD, Cassani R, Lottenberg AM, Casella Filho A, Araújo DB, Cesena FY, Alves RJ, Fenelon G, Nishioka SAD, Faludi AA, Geloneze B, Scherr C, Kovacs C, Tomazzela C, Carla C, Barrera-Arellano D, Cintra D, Quintão E, Nakandakare ER, Fonseca FAH, Pimentel I, Santos JE, Bertolami MC, Rogero M, Izar MCO, Nakasato M, Damasceno NRT, Maranhão R, Cassani RSL, Perim R, Ramos S. Sociedade Brasileira de Cardiologia. I Diretriz sobre o consumo de Gorduras e Saúde Cardiovascular. Arq Bras Cardiol 2013;100(1 Supl. 3):1-40.

10. Brasil. Ministério da Saúde (MS). Vigitel Brasil 2014: Vigilância de fatores de risco e proteção para doenças crônicas por inquérito telefônico. Brasília: MS; 2015. [acessado 2016 Set 06]. Disponível em: http://bvsms.saude. gov.br/bvs/publicacoes/vigitel_brasil_2014.pdf

11. World Health Organization (WHO). Global status report on noncommunicable diseases 2014. [acessado 2016 Set 07]. Disponível em: http://apps.who.int/iris/bitstream/10665/148114/1/9789241564854_eng.pdf?ua=1

12. Brasil. Ministério da Saúde (MS). Vigitel Brasil 2007: Vigilância de fatores de risco e proteção para doenças crônicas por inquérito telefônico. Brasília: MS; 2008. 136 p. [acessado 2016 Set 06]. Disponível em: http://bvsms. saude.gov.br/bvs/publicacoes/vigilancia_risco_doencas_inquerito_telefonico_2007.pdf

13. Kim HJ, Fay MP, Feuer EJ, Midthune DN. Permutation tests for joinpoint regression with applications to cancer rates. Stat Med 2000; 19(3):335-351.

14. Gardner MJ, Altman DG. Confidence intervals rather than P values: estimation rather than hypothesis testing. Br Med J (Clin Res Ed.) 1986; 292(6522):746-750.

15. Mondini L, Monteiro CA. Mudanças no padrão de alimentação da população urbana brasileira (1962-1988). Rev Saude Publica 1994; 28(6):433-439

16. Marinho MCS, Hamann EM, Lima ACCF. Práticas e mudanças no comportamento alimentar na população de Brasília, Distrito Federal, Brasil. Rev Brasileira de Saúde Materno Infantil 2007; 7(3):251-261.

17. Brasil. Ministério da Saúde (MS). Inquérito domiciliar sobre comportamentos de risco e morbidade referida de doenças e agravos não transmissíveis: Brasil, 15 capitais e Distrito Federal, 2002-2003. Rio de Janeiro: INCA; 2004.

18. Claro RM, Santos MAS, Oliveira TP, Pereira CA, Szwarcwald CL, Malta DC. Consumo de alimentos não saudáveis relacionados a doenças crônicas não transmissíveis no Brasil: Pesquisa Nacional de Saúde, 2013. Epidemiol. Serv. Saúde 2015; 24(2):257-265. 
19. Acebrón LB, Dopico DC. The importance of intrinsic and extrinsic cuesto expected and experienced quality: An empirical application for beef. Food Qual Prefer 2000; 11(3):229-238.

20. Realini CE, Kallas Z, Pérez-Juan M, Gómez I, Olleta JL, Beriain MJ, Alberti P, Sañudo C. Relative importance of cues underlying Spanish consumers' beef choice and segmentation, and consumer liking of beef enriched with n-3 and CLA fatty acids. Food Qual Prefer 2014; 33:74-85.

21. Ngapo TM, Dransfield E. British consumers preferred fatness levels in beef: Surveys from 1955, 1982 and 2002. Food Qual Prefer 2006; 17(5):412-417.

22. Henchion M, McCarthyb M, Resconi VC, Troy D. Meat consumption: Trends and quality matters. Meat Sci 2014; 98(3):561-568.

23. Mathijs E. Exploring future patterns of meat consumption. Meat Sci 2015; 109:112-116.

24. Popkin BM. The World Is Fat--The Fads, Trends, Policies, and Products That Are Fattening the Human Race. New York: Avery-Penguin Group; 2008.

25. Schneider BC, Duro SWS, Assunção MCF. Consumo de carnes por adultos do sul do Brasil: um estudo de base populacional. Cien Saude Colet 2014; 19(8):3583-3592.

26. Chambers S, Lobb A, Butler LT, Traill WB. The influence of age and gender on food choice: a focus group exploration. International Journal of Consumer Studies 2008; 32(4):356-365.

27. Frenk J, Frejka T, Bobadilha JL, Stern C, Lozano R, Sepúlveda J, José M. La transición epidemiológica em América Latina. Bol of Sanit Panam 1991; 111(6):485496.

28. Duarte EC, Barreto SM. Transição demográfica e epidemiológica: a Epidemiologia e Serviços de Saúde revisita e atualiza o tema. Epidemiol Serv Saúde 2012; 21(4):529-532.

29. Instituto de Pesquisa Econômica Aplicada (IPEA). Secretaria de Planejamento e Investimentos Estratégicos (SPI). Objetivos de Desenvolvimento do Milênio: Relatório Nacional de Acompanhamento. Brasília: Ipea, MP, SPI; 2014.

30. Kappel DB. Índice de desenvolvimento infantil no Brasil: Uma análise regional. Revista Brasileira de Educação 2007; 12(35):232-240.

31. Instituto Brasileiro de Geografia e Estatística (IBGE). Pesquisa de Orçamentos Familiares 2008-2009: Despesas, Rendimentos e Condições de Vida. Rio de Janeiro: IBGE; 2010.

32. Levy-Costa RB, Sichieri R, Pontes NS, Monteiro CA. Disponibilidade domiciliar de alimentos no Brasil: distribuição e evolução (1974-2003). Rev Saude Publica 2005; 39(4):530-540.

33. Iser BPM, Claro RM, Moura EC, Malta DC, Neto OLM. Fatores de risco e proteção para doenças crônicas não transmissíveis obtidos por inquérito telefônico - Vigitel Brasil - 2009. Rev Bras Epidemiol 2011; 14(Supl. 1):90-102

34. Azevedo ECC, Dias FMRS, Diniz AS, Cabral PC. Consumo alimentar de risco e proteção para as doenças crônicas não transmissíveis e sua associação com a gordura corporal: um estudo com funcionários da área de saúde de uma universidade pública de Recife (PE), Brasil. Cien Saude Colet 2014; 19(5):1613-1622.
35. Lenz A, Olinto MTA, Dias-da-Costa JS, Alves AL, Balbinotti M, Pattussi MP, Bassani DG. Socioeconomic, demographic and lifestyle factors associated with dietary patterns of women living in Southern Brazil. Cad Saude Publica 2009; 25(6):1297-1306.

36. Park SY, Murphy SP, Wilkens LR, Yamamoto JF, Sharma S, Hankin JH, Henderson BE, Kolonel LN. Dietary patterns using the food guide pyramid groups are associated with sociodemographic and lifestyle factors: The Multiethnic Cohort Study. J Nutr 2005; 135(4):843849.

37. Perrin AE, Dallongeville J, Ducimetiere P, Ruidav- ets JB, Schlienger JL, Arveiler D, Simon C. Interations between traditional regional determinants and socio-economic status on dietary patterns in a sample of French men. Br J Nutr 2005; 93(1):109-114.

38. World Health Organization (WHO). Preventing chronic diseases: a vital investment. Geneva: WHO; 2005.

39. Westhoek H, Lesschen JP, Rood T, Wagner S, De Marco A, Murphy-Bokern D, Leip A, Grinsven H, Sutton MA, Oenema O. Food choices, health and environment: Effects of cutting Europe's meat and dairy intake. Global Environmental Change 26 2014:196-205.

40. Godfray HCJ, Beddington JR, Crute IR, Haddad L, Lawrence D, Muir JF, Pretty J, Robinson S, Thomas SM, Toulmin C. Food security: the challenge of feeding 9 billion people. Science 2010; 327(5967):812-818.

41. Food and Agriculture Organization of the United Nations (FAO). World agriculture: towards 2030/2050: prospects for food, nutrition, agriculture and major commodity groups. FAO, Rome. 2006. [acessado 2016 Set 07]. Disponível em: www.fao.org/docrep/010/a0701e/ a0701e00.HTM

42. Brasil. Ministério da Saúde (MS). Plano de ações estratégicas para o enfrentamento das doenças crônicas não transmissiveis (DCNT) no Brasil 2011-2022. Brasília: MS; 2011.

43. Monteiro CA, Moura EC, Jaime PC, Claro RM. Validade de indicadores do consume de alimentos e bebidas obtidos por inquérito telefônico. Rev Saude Publica 2008; 42(4):582-589.

44. Mendes LL, Campos SF, Malta DC, Bernal RTI, Sá NNB, Velásquez-Meléndez G. Validade e reprodutibilidade de marcadores do consumo de alimentos e bebidas de um inquérito telefônico realizado na cidade de Belo Horizonte (MG), Brasil. Rev Bras Epidemiol 2011;14(1):80-89.

45. Bernal R, Silva NN. Cobertura de linhas telefônicas residenciais e vícios potenciais em estudos epidemiológicos. Rev Saude Publica 2009; 43(3):421-426.

Artigo apresentado em 07/10/2016

Aprovado em 24/06/2017

Versão final apresentada em 26/06/2017 